\title{
Calibration method for direct conversion receiver front-ends
}

\author{
R. Müller and H.-J. Jentschel \\ Institute of Traffic Communications Engineering, Dresden University of Technology, Germany
}

\begin{abstract}
Technology induced process tolerances in analog circuits cause device characteristics different from specification. For direct conversion receiver front-ends a system level calibration method is presented. The malfunctions of the devices are compensated by tuning dominant circuit parameters. Thereto optimization techniques are applied which use measurement values and special evaluation functions.
\end{abstract}

\section{Introduction}

Modern communication circuits are realized as integrated circuits. Because of process tolerances there are both defective and non-defective components produced. Test and calibration intend the separation of defective from non-defective modules as well as increasing yield. This paper presents a calibration method for direct conversion receiver (DCR) front-ends as device under test (DUT). The DCR front-end consists of a number of subsystems. These subsystems, e.g. mixer, LNA, filters, are called blocks and realize different functions. Basically, the DUT has a chain structure. The signal paths from the antenna to the A/D-converter corresponds to cascades of blocks. Therefore, the effects of the blocks on the signal superpose because the signal has to pass all blocks arranged in the signal path. From this follows, that conclusions can be given about the overall characteristic of the whole system using the output signal in relation to the input signal. Such analysis of the characteristic of an entire system is called system level test.

In reality, the implementation of such a system level test in combination with a system calibration is associated with some problems. There are evaluable characteristic system quantities needed to calibrate the system. These values can be obtained using special test signals. However, the nature of the measured information is restricted. There are only output signals available. Measuring points inside the DUT are not assumed. The analysis of the output signal should result in system values of the DUT, which can be compared to the specification or to the characteristic system quantities of a golden device. The problem in this context is as follows: it is possible to characterize enough the functionality of the DUT in respect of the requirements of the specifications using measured information of restricted nature. The integration of calibration mechanisms into the DUT requires adjustability of the block parameters. So the characteristic quantities of the blocks can be changed. Practically, block parameters correspond to circuit elements, e.g. resistors, capacitors.

It is clear from different reason, that it is impossible to make changeable all circuit elements in all blocks. Only a limited number of elements, the so called dominant parameters, can be prepared for tuning. Moreover, these parameters can be changed in a limited range only. So there are two problems in this context. First, the dominant parameters parameters must be selected taking in consideration practicability and costs. Second, it is possible to get desired changes of the DUT's output behavior by tuning a limited number of predetermined circuit elements.

At last there is the problem, that tuning of one parameter is affecting all system quantities on the output (Rühle, 2002). Because of this reason the use of optimization algorithms is proposed to correct the characteristic system quantities of the DUT. The dominant parameters are realized as variable parameters with a limited range. The optimization results in values for the dominant parameters for the correction of the DUT. In entirety the signal generator, signal analysis and optimization form a calibration at system level.

Instead of real circuits mathematical block models are considered to simplify the investigations. The feasibility of the calibration method can be verified with these models in principle. Malfunctions of the DUT are assigned to specific blocks and are included in the model.

\section{Correspondence to: R. Müller}

(reik.mueller@tu-dresden.de) 


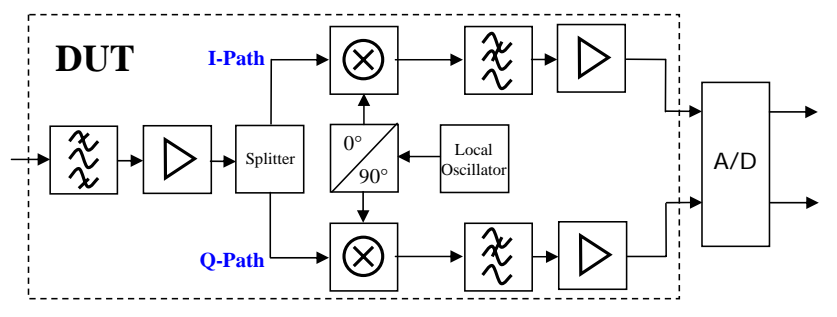

Fig. 1. The direct conversion receiver as test object.

\section{The DCR as DUT}

In our case a direct conversion receiver front-end is used as a DUT. Its block structure is shown in Fig. 1. The input block is the system filter (band pass filter). It is followed by a LNA. After pre-amplification the signal is split and fed to the Iand Q-path. The following mixers are driven by $90^{\circ}$ phase shifted oscillator signals. The subsequent blocks in the chain are a low pass filter and a baseband amplifier in every path. The low pass filter and the amplifier often are applied as an active filter. These blocks determine the system characteristics, superpose in their influence on these characteristics and are responsible for functionality, properties and possible malfunctions of the DUT. But the influence on the functionality of the DUT is different per block (see Sect. 3). From this reason variable block properties can be used to compensate process tolerances.

\section{The model of the DCR}

A DCR front-end is a complex structure with a large number of devices. It is not possible to correct the value of all devices, because design effort and the need of chip area would be too high. Additionally a highly dimensional parametric space would result, which forms the basis of the correction of characteristic system values and can be applied only with high CPU-intensive computations and high effort of time. The latter is a problem of circuit design and the associated search for optimal variants of circuits (Müller et al., 2006). Because significant values have to be estimated by measurements and the measurements are time consuming, it is necessary to consider only dominant parameters for changing the DUT-properties. With such parameters the properties of the device can be changed efficiently, because there is a high sensitivity of the target values (Sect. 5) with reference to the variable device values. Such relations have to be analyzed by an sensitivity analysis of the whole circuit (Pursche, 2005; Hamida and Kaminska, 1993).

In the following it is assumed, that a limited number of tunable parameters has been chosen. The task is to correct the degradation of system performance caused by deviations in all parameters by this limited number of variable parameters, until the measurements of the DUT are possibly close

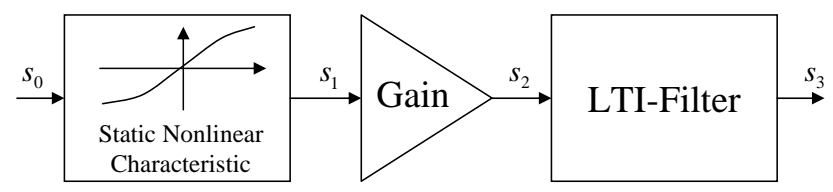

Fig. 2. Hammerstein model of the DUT.

to the measurements of the reference. The accuracy of the measurements is not regarded. The assumption is, that the measurement values are estimated faultlessly.

To achieve an easier verification of the proposed method the model has been kept simple consciously. The model only describes one path of the receiver front-end. One signal path of the DUT consists of the system filter (band pass filter, wide band), LNA, splitter, mixer, baseband filter (low pass filter) and a baseband amplifier (Fig. 1). The frequency shift of the mixing process has been considered as ideal, so it is not regarded in the model. The filter characteristics of all blocks have been summarized in a passive 2 nd order Butterworth filter. Because the bandwidth of the system filter is much greater than that of the baseband filter, the cut-off frequency of the band pass filter has not to be considered. The splitter is only intended to affect the signal amplitude. The amplification of the active blocks superposes with the attenuation of the other blocks. The resulting amplification/attenuation is combined in an ideal amplification block with the amplification $v$. The nonlinearities of mixer, LNA and baseband amplifier are modeled by a static nonlinear characteristic. The analog to digital converter is considered as ideal. The final model is a Hammerstein model as shown in Fig. 2.

To show the relation between values of devices (e.g. capacitances) and the parameters of the model, the low pass filter has been realized as transfer function which includes device values. The transfer function of the low pass filter of the model is:

$G(s)=\left[2+K_{1} s+K_{2} s^{2}+K_{3} s^{3}+K_{4} s^{4}\right]^{-1}$

with

$K_{1}=R C_{2}+R C_{1}+\left(L_{1}+L_{2}\right) / R$

$K_{2}=\left(C_{1}+C_{2}\right)\left(L_{1}+L_{2}\right)$

$K_{3}=C_{1} L_{2}\left(R C_{2}+L 1 / R\right)$

$K_{4}=C_{1} C_{2} L_{1} L_{2}$

The static nonlinear characteristic is modeled by:

$s_{1}=\theta\left(s_{0}\right)=c \times \tanh \left(\frac{s_{0}+A P}{c}\right)-c \times \tanh \left(\frac{A P}{c}\right)$

and realizes the operation point dependent characteristic of a differential amplifier. The parameter $A P$ represents the operation point and is a variable parameter, $s_{0}=s_{0}(t)$ is the input signal in time domain and the parameter $c$ stretches the characteristic to regulate the "strength" of the nonlinearity. 
With the model in Fig. 2 the output signal $s_{3}(t)$ in dependence of the input signal $s_{0}(t)$ is:

$s_{3}(t)=\int_{-\infty}^{\infty} g(\tau) \times s_{2}(t-\tau) \mathrm{d} \tau$

with $g(\tau)$ as impulse response according to Eq. 1. The filter input signal $s_{2}(t)$ results from Eq. 6 and the linear amplification $v$ :

$s_{2}(t)=v \times \theta\left(s_{0}(t)\right)$

\section{Measurement and correction}

The correction structure is shown in Fig. 3. For correction the present state of the DUT has to be estimated whether the specification is matched or not. A spectrum analysis is applied to the sampled output signal of the receiver. From the spectrum the following DUT parameters are estimated: cutoff frequency, over-all attenuation, IP3, I/Q-imbalance and frequency deviation of the oscillator signal. The extraction of these parameters is described in Lupea et al. (2003); Müller and Jentschel (2006a); Müller and Jentschel (2006b); DETAILS (2005). Using conventional measurement methods requires multiple measurements to estimate all these parameters (Kundert, 2002; Schaub and Kelly, 2004).

The evaluation of the measurement values includes a comparison with the specification and an optimization, described in the following sections. The evaluation results in parameters $x_{v}$, which are numerical values and represent the new state of the variable circuit elements/block parameters. The control unit decodes the numerical parameters $x_{v}$ and transforms them into physical circuit values or operation point currents or voltages. Technical realizations are VGAs, varactors, fractional-N-PLLs, switched capacitors and switchable resistor and capacitor networks (Csipkes, 2006).

Subsequent the state of the DUT with the new parameter settings is estimated in relation to the specification again.

\section{The objective function}

The chain of DUT, measurement facilities and application of the control values realizes a function which function values are represented by measurement values. Every measurement is a calculation of a value of a function. A change in circuit device values changes the function. According to Fig. 3 the arguments of this function are represented by the parameters $x_{v}$. It is convenient to summarize these parameters in a parameters vector.

$\mathbf{x}=\left[x_{1} \ldots x_{k}\right]^{\mathrm{T}}$

Every parameter $x_{v}$ can be set on discrete values between 0 and maximum register value $x_{v, \max }$ (control word for a device parameter). Dependent on the controlled device value

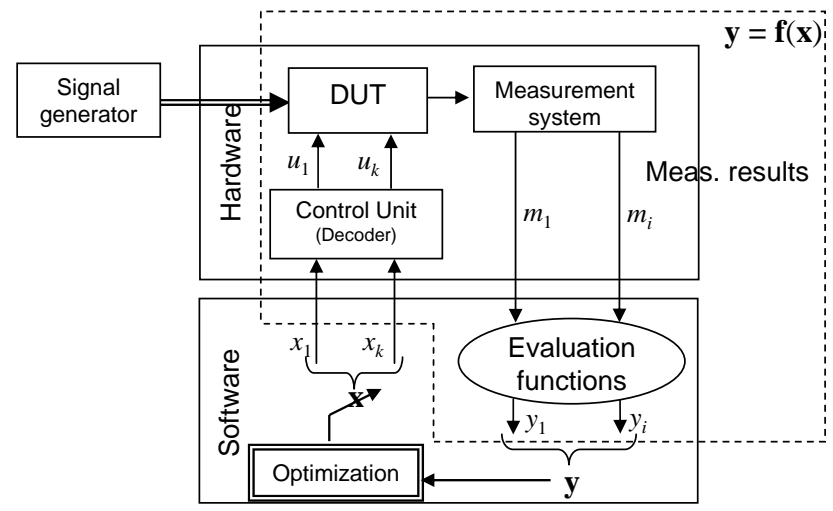

Fig. 3. Structure of the adjustment arrangement and scheme of the functional relation between parameter vector and target vector.

like capacitances or resistances the parameters $x_{v}$ are converted in different physical values. The relationship between the parameters and the physical values is represented by functions $g_{v}$.

$\mathbf{u}=\left[g_{1}(x) \ldots g_{k}(x)\right]^{\mathrm{T}}=\mathbf{g}(\mathbf{x})$

The linear relation between $u_{v}$ and $x_{v}$ is:

$u_{v}=u_{v, \min }+\left(u_{v, \max }-u_{v, \min }\right) \times x_{v} / x_{v, \max }$

and is for example valid for switchable resistor and capacitor networks or linear adjustable gain. There are also nonlinear relations like the capacitance control of varactors. The linear change of the control voltage of a varactor in conformity to Eq. 11 the capacitance changes in nonlinear way (Tietze and Schenk, 1990, p. 27).

With different settings $u_{v}$ of the circuit device values the input signal is transformed into an output signal $s_{3}(t)=s_{3}\left(s_{0}(t), \mathbf{u}\right)$ in different way. The measurement of the output signal $s_{3}(t)$ results in measurement values $m_{\mu}$. Hence, there is a relation between output signal $s_{3}(t)$ and measurement values. Therewith there is also a relation between circuit device values $u_{v}$ and the measurement values. Because of the manifold and complicated functional relations between output signal and measurement values, only the formal context is given:

$\mathbf{m}=\left[m_{1} \ldots m_{i}\right]^{\mathrm{T}}=\left[h_{1}(\mathbf{u}) \ldots h_{i}(\mathbf{u})\right]^{\mathrm{T}}=\mathbf{h}(\mathbf{u})$

Measurement values are for example IP3, amplitude characteristic, NF, power loss, etc. The measurement values have to be adapted to the expected values of the optimization algorithm. The optimization goal is the maximum or the minimum of a function. As a result the measurement values $m_{i}$ have to be transformed in an appropriate manner by evaluation functions:

$\mathbf{y}=\left[y_{1} \ldots y_{i}\right]^{\mathrm{T}}=\left[\zeta_{1}(\mathbf{m}) \ldots \zeta_{i}(\mathbf{m})\right]^{\mathrm{T}}=\zeta(\mathbf{m})$ 


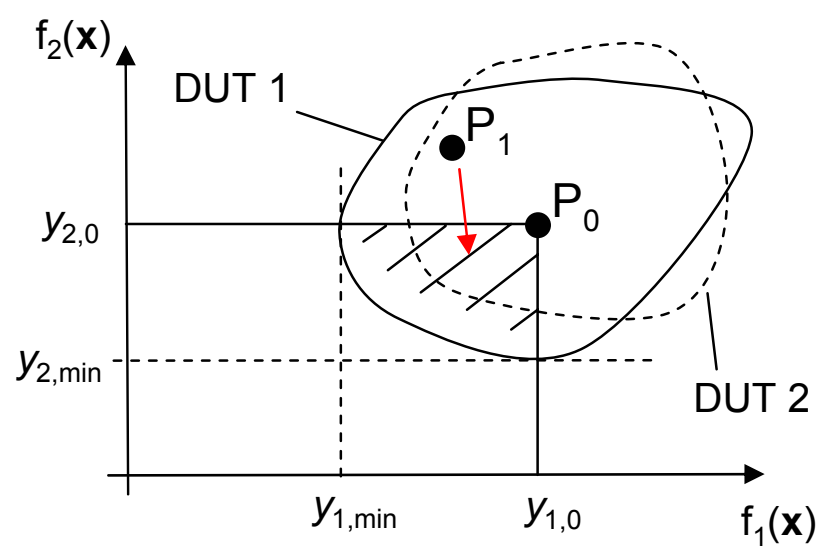

Fig. 4. Co-domain of two different DUT.

The transformation in target values has to be applied to all measurement values and is examined in Sect. 7 more detailed. Because there are several measurement values, a multi-criteria optimization problem occurs. With Eq. 9 to Eq. 12 and Eq. 13 the vectorial objective function follows formally (cp. Fig. 3):

$\mathbf{y}=\mathbf{f}(\mathbf{x})$

Therewith the general relation between parameters of the DUT and objective function is shown. As it can be seen from Eq. 7 and from the description of Eq. 12, the objective functions $f_{\mu}(\mathbf{x})$ consists of complex analytical expressions and are difficult to present. That is why these relations are not specified in this paper.

\section{Range of values}

Equation 14 has a limited co-domain, because every scalar parameter $x_{v}$ is variable only in an limited range. There is no point accessible outside the limited range, even with arbitrary settings of $\mathbf{x}$. Because of process tolerances in circuit device values different DUT have co-domains which are differently limited. Using Eq. 14 the general formulation of the optimization task is:

$$
\min _{\mathbf{x}} \mathbf{f}(\mathbf{x})
$$

Assuming the DUT is conform to the specification in any valid parameter combination, $P_{0}$ marks that point in the codomain, where the minimum requirements of specification are sufficiently reached (Fig. 4). The evaluation functions $\zeta_{i}$ (cp. Sect. 7) ensure the minimum of a target value $y_{i}$ is reached, when the appropriate property of the DUT is best possible. Hence, all points left and below the point $P_{0}$ represents a DUT that meets the specification. The hatched area in Fig. 4 shows that case for a two-dimensional co-domain. Consequently the calibration of the DUT is simplified to the task to shift the current point of state into the hatched area. If that condition has been achieved, the optimization can be finished. Thus, a search for the best point is not necessary and the requirements for optimization are simplified.

The indicator for compliance with the specification is examined in the following. The vector $\mathbf{r}_{0}$ from origin of ordinates to the point $P_{0}$ is calculated from specification and evaluation functions $\zeta_{i}$. The point $P_{1}$ represents the current point of state with the position vector $\mathbf{r}_{1}$. From the point $P_{0}$ to the current point of state points the vector:

$\mathbf{r}_{\mathrm{D}}=\left[d_{1} \ldots d_{i}\right]^{\mathrm{T}}=\mathbf{r}_{1}-\mathbf{r}_{0}=\left[\begin{array}{c}y_{1,1}-y_{1,0} \\ \vdots \\ y_{i, 1}-y_{i, 0}\end{array}\right]$

in the $i$-dimensional co-domain of the objective function. Where $y_{\mu, \nu}$ is the $\mu$-th component of the vector $v$. The exit condition is

$d_{v} \leq 0 \quad$ with $\quad v=1 \ldots i$

If this condition is complied, the value of the penalty function is 0 , else it is

$f_{\text {straf }}=\sum d_{v}$ with $\quad \forall d_{v}>0$

Hence, the goal of calibration is, that the value of the penalty function reaches 0 . Then the DUT meets the specification.

\section{Evaluation functions}

In the following examples are given how to calculate values of the objective function from measurement values.

The intermodulation point (IP) is a value to rate nonlinear distortions. Mostly in communications there is an interest for linearity. I.e. an IP which is going towards infinity is wanted. To formulate a task of minimization with the $3 \mathrm{rd}$ order intermodulation point (IP3) the conversion from IP3 to a value of the objective function is:

$y_{\mathrm{IIP}}=\zeta_{1}\left(\mathrm{IIP}_{3}\right)=-c_{1} \times \mathrm{IIP}_{3}$

From the value of the specification accordingly follows:

$y_{\mathrm{IIP}_{3}, 0}=\zeta_{1}\left(\mathrm{IIP}_{3, \min }\right)=-c_{1} \times \mathrm{IIP}_{3, \min }$

where $\mathrm{IIP}_{3 \min }$ is the minimum acceptable IP3 according to the specification.

For rating the accuracy of the frequency of the local oscillator on chip, the frequency $f_{\mathrm{LO}}$ has to be measured and compared with the target frequency $f_{\mathrm{LO}, 0}$.

$y_{\mathrm{LO}}=\zeta_{2}\left(f_{\mathrm{LO}}\right)=-c_{2} \times\left(f_{\mathrm{LO}}-f_{\mathrm{LO}, 0}\right)^{2}$

The bound of $y_{\mathrm{LO}}$ pursuant to the specification is

$$
\begin{aligned}
y_{\mathrm{LO}, 0} & =-c_{2} \times\left[\left(f_{\mathrm{LO}, 0} \pm \Delta f\right)-f_{\mathrm{LO}, 0}\right]^{2} \\
& =-c_{2} \times \Delta f^{2}
\end{aligned}
$$


where $\Delta f$ is the acceptable frequency deviation from the target frequency. For rating the constancy of the amplitude characteristic in the pass band of the DUT the spectrum of the output signal has to be estimated. At this, samples in frequency domain with an determined fundamental frequency occurs. With that samples and the input signal the transfer characteristic $\left|H\left(f_{v}\right)\right|$ can be estimated approximately. The sum of the square error of the transfer factors with reference to a constant $H_{\text {ref }}$ (mean value or a reference) is a figure of merit of the waviness of the amplitude characteristic:

$y_{\mathrm{Ampl}}=\zeta_{3}(H)=\sum_{\nu=\nu_{\min }}^{\nu_{\max }}\left(\left|H\left(f_{\nu}\right)\right|-\left|H_{\mathrm{ref}}\right|\right)^{2}$

with the bound $y_{\mathrm{Ampl}, 0}=\mathrm{const}$

The presented options for calculating values of the objective function from measurement values are also applicable for conversion of a number of further measurement values: I/Q-mismatch, cut-off frequency, signal power, power consumption, noise figure, etc.

So, the vector of the objective function can be written as:

$\mathbf{r}_{1}=\left[\begin{array}{c}y_{1,1} \\ y_{2,1} \\ y_{3,1} \\ \vdots\end{array}\right]=\left[\begin{array}{c}y_{\mathrm{IIP} 3} \\ y_{\mathrm{LO}} \\ y_{\mathrm{Ampl}} \\ \vdots\end{array}\right]$

The vector $\mathbf{r}_{0}$ has to be created from the acceptable bounds in equivalence to $\mathbf{r}_{1}$. Thereupon the value of the penalty function is calculated like shown in Sect. 6.

\section{Verification of the calibration method}

For verification of the feasibility of the calibration method, simulations with the model from Sect. 3 have been realized exemplarily. As reference model/golden device the following model parameters has been chosen: $C_{1}=58.781 \mu \mathrm{F}$, $C_{2}=24.348 \mu \mathrm{F}, L_{1}=60.087 \mu \mathrm{H}, L_{2}=146.95 \mu \mathrm{H}$, reference resistance $R=50 \Omega, v=1, A P=0$ and $c=10$. The input signal is a multi-tone signal with 10 harmonics with the lowest frequency at $10 \mathrm{kHz}$, the highest frequency at $110 \mathrm{kHz}$ and spectral gap between the lower and the higher harmonics. The amplitude of every harmonic is $0 \mathrm{~dB}$. The frequency distance (fundamental frequency) between the harmonics is $10 \mathrm{kHz}$. The spectrum of the output signal is calculated. The complex amplitudes of the output signal at these frequency bins, which are occupied by the harmonics in the input signal, are written as a vector $\mathbf{v}$. From this vector the $\mathrm{IIP}_{3}$ is calculated using the multi-tone test method described in DETAILS (2005). Additionally the power level $p_{3 \mathrm{~dB}}$ at the intended cut-off frequency and the mean signal power level $\bar{p}$ are calculated and compared with the specification. Hence, the values of the objective function are $f_{\mathrm{IIP}_{3}}, f_{\bar{p}}$ and $f_{p_{3 \mathrm{~dB}}}$. Because of using a Butterworth filter, it can be assumed that there is linearity in the pass band. Because the oscillator frequency is not considered in the model, the application of Eq. 21 and Eq. 22 has been dropped.

The variable parameters of the DUT are $u_{1}=C_{1}, u_{2}=C_{2}$, $u_{3}=v$ and $u_{4}=A P$. The remaining parameters of the DUT are invariant values. For simplification the parameter vector is considered a continuously variable. The transformation of the parameters $x_{v}$ in circuit device values $u_{v}$ linear functions $g_{v}$ are constructed according to Eq. 11. Two cases has been simulated:

- Case 1: the invariant values of the DUT are equal to that of the reference model.

- Case 2: the invariant values of the DUT have a deviation of $+10 \%$ of the values of the reference model.

The initial values of the variable parameters have a deviation of $-20 \%$ of the reference values in both cases. The initial value of the parameter $A P$ has been set to 9.5. The DUT is stimulated with the same input signal like the reference model. The measurement values are estimated from the output signal of the DUT. The values of the objective functions are calculated according to Sect. 7 and the value of the penalty function according to Eq. 18 .

For simplification of the optimization the optimization task has been reduced using a scalar objective function. The point of state of the golden device is inside the area of matched specification (cp. Fig. 4). Because the values of the objective function are calculated from measurement values and the measurement values are calculated from the output signal, the approximation of the output signal of the DUT to that of the golden device potentially leads to an approximation of the point of state in co-domain. The mean square error $f_{q}$ of the deviation of the output spectrum $\mathbf{v}_{\text {DUT }}$ of the DUT and the output spectrum $\mathbf{v}_{\text {ref }}$ of the reference model is calculated.

$f_{q}(\mathbf{x})=\left|\mathbf{v}_{\text {DUT }}(\mathbf{x})-\mathbf{v}_{\text {ref }}(\mathbf{x})\right|^{2}$

The mean square error is a scalar value that is optimized using Quasi-Newton-method (Matlab, 2007) in terms of the parameter vector $\mathbf{x}$. Doing so, the optimization task according to Eq. 15 is reduced to:

$\min _{\mathbf{x}} f_{q}(\mathbf{x})$

While running the optimization the exit condition according to Eq. 17 or Eq. 18 respective is checked after every iteration step. The optimization is canceled if the specification is matched. The values of the specification has been chosen exemplarily like the parameters of the test object. So there is no direct applicatory significance. The values of specifications are $\mathrm{IIP}_{3}>25.4 \mathrm{~dB}, p_{3 \mathrm{~dB}} \leq 27.87 \mathrm{~dB}$ and $\bar{p}=29.7 \mathrm{~dB}$. The associated values of the DUT and the reference model are listed in Table 1.

Without consideration of the exit condition in case 1 the optimization of the DUT results in the same values of the parameter vector like the values of the reference model after 36 iterations and 210 measurements. With regard to the 
Table 1. Measurement values.

\begin{tabular}{lrrr}
\hline CASE 1 & $\mathrm{IIP}_{3} / \mathrm{dB}$ & $p_{3 \mathrm{~dB}} / \mathrm{dB}$ & $\bar{p} / \mathrm{dB}$ \\
\hline Reference model & 25.711 & 27.870 & 29.777 \\
Unoptimized DUT & 25.095 & 22.107 & 22.858 \\
Spec. reached (exit) & 25.710 & 27.839 & 29.757 \\
Opt. without aborting & 25.711 & 27.870 & 29.777 \\
\hline CASE 2 & $\mathrm{IIP}_{3} / \mathrm{dB}$ & $p_{3 \mathrm{~dB}} / \mathrm{dB}$ & $\bar{p} / \mathrm{dB}$ \\
\hline Reference model & 25.711 & 27.870 & 29.777 \\
Unoptimized DUT & 24.296 & 22.402 & 23.523 \\
Spec. reached (exit) & 26.606 & 27.808 & 29.305 \\
Opt. without aborting & 26.610 & 28.597 & 29.910 \\
\hline
\end{tabular}

specification the goal of correction is already reached after 31 iterations and 185 measurements. In this case the estimated parameters are $C_{1}=58.870 \mu \mathrm{F}, C_{2}=24.337 \mu \mathrm{F}$, $v=0.998$ and $A P=-0.068$.

In case 2 the optimization converges after 36 iterations and 195 measurements. Because the invariant parameters of the DUT are different to the parameters of the reference model, the variable parameters are estimated with $C_{1}=53.933 \mu \mathrm{F}$, $C_{2}=21.617 \mu \mathrm{F}, v=0.990$ and $A P=0.040$. The values of the specification are already achieved after 5 iterations and 30 measurements. At this the variable parameters are estimated with $C_{1}=50.708 \mu \mathrm{F}, C_{2}=22.365 \mu \mathrm{F}, v=0.959$ and $A P=2.311$.

The specification is matched after optimization and applying exit condition. Without applying the exit condition in case 2 the specification is not always matched during the progressive iteration process, i.e. the point of state temporarily leaves the region of matched specification in the co-domain.

\section{Conclusions}

A method to correct static deviations of parameters of DCR front-ends has been presented. Values of selected circuit devices of a DUT which does not match the specification are corrected applying an optimization algorithm until the specification is met. To enable the variability of the values of the selected circuit devices the circuit design has to be extended. Deviations of the values of the circuit devices caused by process tolerances can be compensated that way. Measurements of the DUT provide objective function values needed for optimization. The parameter alignment is done at the DUT directly. The optimization is an iterative method with several measurements at every iteration step. That is why the major disadvantage of this method concerns the measurement time, because it is possible that more than one hundred measurements are needed for correction.
In particular the noise increases the number of iteration steps which are needed. Additionally the duration of measurement has to be increased to average over time. With noise the accuracy of the measurement values decreases, which cause convergence problems.

Acknowledgements. This work has been supported by the German Government (BMBF). Additionally this work has been supported by Infineon Technologies AG.

\section{References}

Csipkes, G.-L.: Integrated realizations of reconfigurable low pass and band pass filters for wide band multi-mode receivers, Dissertation TU-Dresden, 2006.

DETAILS: Milestone Report M15-AP1.3 - Calibration and Test Friendly Design, BMBF project "DETAILS" Grant 01M3071, Milestone report 2005, 43-61, 2005.

Hamida, N. B. and Kaminska, B.: Multiple Fault Analog Circuit Testing by Sensitivity Analysis, J. Electron. Test., 4, 331-343, 1993.

Kundert, K. S.: Accurate and Rapid Measurement of $I P_{2}$ and $I P_{3}$, Designer's Guide Consulting, Inc., http://www.designers-guide. org, 2002.

Lupea, D., Pursche, U., and Jentschel, H.-J.: RF-BIST: Loopback Spectral Signature Analysis. Proc. of Design, Automation and Test in Europe Conference - DATE 03, Munich, March 3-7 2003, 478-483, 2003.

Müller, R. and Jentschel, H.-J.: Testmethode für Direct Conversion Receiver, Analog'06, ITG Fachbericht 196, 95-100, 2006 a.

Müller, R. and Jentschel, H.-J.: A Built-In-Self-Test view on estimation of mixer-parameters of direct conversion receiver front-ends, 18. ITG/GI/GMM Workshop "Testmethoden und Zuverlässigkeit von Schaltungen und Systemen”, Titisee, 12-14 März 2006, 71-75, 2006b.

Müller, D., Gräb, H., and Schlichtmann, U.: Optimierung analoger Schaltungsblöcke mittels Pareto-Wellenfront-Optimierung, Analog'06, ITG Fachbericht 196, 33-38, 2006.

Pursche, U.: Spektrale Signalflussmodellierung durch Harmonischen-Transfer-Matrizen für den Selbsttest und die Selbstkorrektur von Hochfrequenzschaltungen. Dissertation TU-Dresden, ISBN 3-8325-0996-8, 162-168, 2005.

Rühle, Th.: Entwurfsmethodik für Funkempfänger, Dissertation TU-Dresden, 46-56, 2002.

Schaub, K. B. and Kelly, J.: Production Testing of RF and Systemon-a-Chip Devices for Wireless Communications, ARTECH HOUSE, Inc., ISBN 1-58053-692-1, 2004.

The MathWorks, Inc.: Matlab Optimization Toolbox User's Guide, Sep. 2007, www.mathworks.com, 2007.

Tietze, U. and Schenk, Ch.: Halbleiter-Schaltungstechnik, 9. Auflage, Springer-Verlag Berlin, ISBN 3-540-19475-4, 1990. 